\title{
High-Performance Ultraviolet Light Detection Using Nano-Scale-Fin Isolation AlGaN/GaN Heterostructures with $\mathrm{ZnO}$ Nanorods
}

\author{
Fasihullah Khan $\mathbb{D}$, Waqar Khan $\mathbb{D}$ and Sam-Dong Kim * \\ Division of Electronics and Electrical Engineering, Dongguk University, Seoul 100-715, Korea; \\ fasihullah.khan@dongguk.edu (F.K.); waqarkyz@gmail.com (W.K.) \\ * Correspondence: samdong@dongguk.edu; Tel.: +822-2260-3800
}

Received: 24 February 2019; Accepted: 8 March 2019; Published: 15 March 2019

check for updates

\begin{abstract}
Owing to their intrinsic wide bandgap properties $\mathrm{ZnO}$ and $\mathrm{GaN}$ materials are widely used for fabricating passive-type visible-blind ultraviolet (UV) photodetectors (PDs). However, most of these PDs have a very low spectral responsivity $R$, which is not sufficient for detecting very low-level UV signals. We demonstrate an active type UV PD with a $\mathrm{ZnO}$ nanorod (NR) structure for the floating gate of $\mathrm{AlGaN} / \mathrm{GaN}$ high electron mobility transistor (HEMT), where the AlGaN/GaN epitaxial layers are isolated by the nano-scale fins (NFIs) of two different fin widths (70 and $80 \mathrm{~nm}$ ). In the dark condition, oxygen adsorbed at the surface of the $\mathrm{ZnO}$ NRs generates negative gate potential. Upon UV light illumination, the negative charge on the $\mathrm{ZnO}$ NRs is reduced due to desorption of oxygen, and this reversible process controls the source-drain carrier transport property of HEMT based PDs. The NFI PDs of a $70 \mathrm{~nm}$ fin width show the highest $R$ of a $\sim 3.2 \times 10^{7} \mathrm{~A} / \mathrm{W}$ at $340 \mathrm{~nm}$ wavelength among the solid-state UV PDs reported to date. We also compare the performances of NFI PDs with those of conventional mesa isolation (MI, $40 \times 100 \mu \mathrm{m}^{2}$ ). NFI devices show $\sim 100$ times enhanced $\boldsymbol{R}$ and on-off current ratio than those of MI devices. Due to the volume effect of the small active region, a much faster response speed (rise-up and fall-off times of 0.21 and $1.05 \mathrm{~s}$ ) is also obtained from the NFI PDs with a $70 \mathrm{~nm}$ fin width upon the UV on-off transient.
\end{abstract}

Keywords: high-responsivity; ultraviolet photodetectors; nano-scale fin isolation; wide-band gap semiconductors; $\mathrm{ZnO}$ nanorods; two-dimensional electron gas; visible-blind

\section{Introduction}

Ultraviolet (UV) light detection with high responsivity is of great interest, due to its promising applications in pathology [1], water treatment, safety, and defense-technologies [2]. In most commercialized systems, UV detection has been realized by photomultiplier tubes (PMTs), thermal detectors, Si or Ge based photodetectors (PDs), and charge-coupled devices (CCDs). However, these detection methods pose various problems for practical applications. For example, PMTs exhibit fragile vacuum-tube construction and require a high-voltage bias. Moreover, PMTs are sensitive to the magnetic-field; therefore, they need to be shielded from ambient light. Si based PDs or CCDs are one of the most commonly used solutions, due to their highly matured process technology [3]. Recently, $\mathrm{n}-\mathrm{Si} / \mathrm{p}^{+}-\mathrm{B}$ photodiodes of a very high sensitivity and stability have been demonstrated, but they still exhibit responsivity in the order of $\sim 10^{-1} \mathrm{~A} / \mathrm{W}$ [4]. Many attempts have been made to improve the limited responsivity of Si PDs working in linear mode. Among them, avalanche-mode PDs are now very popular, and single-photon avalanche diodes are also used to obtain high-gain responsivity [5]. However, the use of stop-band filters for visible light reduces the quantum efficiency of these devices in the UV range. 
In recent years, the nanostructures of wide bandgap (WBG) materials, such as diamond, $\mathrm{ZnO}$, III-nitride, and SiC based UV PDs have attracted tremendous research interest for their many advantages [6]. For instance, they are capable of room temperature (RT) operation and have intrinsic optical transparency (visibly-blindness) in the visible spectral range. Furthermore, they have a low thermal conductivity, high breakdown field, and stability at elevated temperatures. In spite of these promising advantages, the WBG semiconductors have very low electron mobility. Even though noble heterostructures with a very high electron mobility of $\sim 10^{6} \mathrm{~cm}^{2} / \mathrm{V} \cdot \mathrm{s}$ using a material system such as $\mathrm{MgZnO} / \mathrm{ZnO}$ [7] have been reported, most of the passive PDs fabricated using conventional WBG semiconductors have very low spectral responsivity [8]. Moreover, the response speeds of PDs based on $\mathrm{ZnO}$ or $\mathrm{GaN}$ are very slow in general because the photoresponse characteristics depend on the well-known bottle-neck chemisorption process of oxygen at the surface of such materials [9].

Zinc oxide ( $\mathrm{ZnO}$ ) nanorod (NR) based UV PDs [10-15] have shown promising results in terms of response speed and spectral responsivity [16]. Significant research effort has been made to improve the performance of the PDs, either by improving the crystalline quality of NRs or by utilizing the composite coaxial structure of $\mathrm{ZnO}$ with other materials, such as copper oxide [17] and graphene [18]. However, most of the passive type PDs reported to date exhibit very slow response speed (tens of seconds) and low responsivity ( hundreds of A/W). Recently, an active-type UV PD using the ZnO NR-gated AlGaN/GaN high electron mobility transistor (HEMT) structure has been attempted as a part of an effort to attain a breakthrough in responsivity $\left(\sim 10^{5} \mathrm{~A} / \mathrm{W}\right)$ and to obtain a relatively fast response speed [19]. However, due to the very high dark current of the device, the HEMT based PD showed a relatively low on-off current ratio of $\sim 3$.

In this study, we demonstrate high-responsivity UV PDs based on the ZnO NR-gated AlGaN/GaN HEMT structure with nano-scale fin isolation (NFI). ZnO NRs act as the floating gate while the UV driven chemisorption process of oxygen at the surface of ZnO NRs [20] controls the conduction of the underlying two-dimensional electron gas (2-DEG) channel. The 2-DEG present at the interface of the AlGaN (barrier) and GaN (channel) layers is due to polarization induced electric potential in the heterostructure [21]. In the NFI PD structure, ZnO NRs surround the channel in the gate area. Consequently, the carriers in the 2-DEG channel are confined along the channel and in perpendicular direction to the interface. Therefore, under dark conditions, the 2-DEG channel is fully depleted due to negative surface potential generated via oxygen adsorption at the surface of $\mathrm{ZnO}$ NRs [9]. This structure resembles enhancement-mode (normally-off) AlGaN/GaN fin-shaped field-effect transistors (FINFETs) [22], demonstrating extremely broad transconductance and excellent off-state characteristics. In this study, the performance of the NFI PDs is compared to conventional mesa isolation (MI) AlGaN/GaN HEMT based PD of $\sim 100 \times 40 \mu \mathrm{m}^{2}$ active area. We examine various photoresponse characteristics of NFI and MI devices and investigate how structural differences influence their performance.

\section{Device Fabrication and Characterization}

Figure 1 shows schematic illustrations of two different HEMT based PD structures examined in this study. The interface of the GaN (channel) and AlGaN (barrier) layers have a confinement of 2-DEG, which acts as channel of very high electron concentration and mobility.

Figure 2 illustrates the essential process steps for our gateless NFI and MI HEMT photodetectors (PDs). The epitaxial layers of AlGaN/GaN were deposited on a 6 inch Si (111) by a metal-organic chemical vapor deposition system at NTT-AT (NTT-Advanced Technology Corporation, Kanagawa, Japan). As shown in the schematics, the undoped GaN buffers and channel layers of 3000-4000 nm thickness were grown first, followed by subsequent growths of the barrier layer of $\sim 20 \mathrm{~nm} \mathrm{Al}_{0.25} \mathrm{GaN}_{0.75}$ and the GaN cap layer of $\sim 1.2 \mathrm{~nm}$. The measured electron sheet carrier concentration and Hall mobility of the epitaxial layer were $\sim 5 \times 10^{12} / \mathrm{cm}^{2}$ and $\sim 1750 \mathrm{~cm}^{2} / \mathrm{V} \cdot \mathrm{s}$, respectively. The active area $\left(100 \times 40 \mu \mathrm{m}^{2}\right)$ for the gateless HEMT was defined by a mesa etching of $100 \mathrm{~nm}$ depth in the case of conventional MI structures, as shown in Figure 1. The NFI structure, on the other hand, was fabricated 
with 10 nano-fin-shaped isolations (10 gate fingers) with the same mesa depth of $100 \mathrm{~nm}$ and two different fin widths $\left(\boldsymbol{W}_{f i n}\right)$ of 70 and $80 \mathrm{~nm}$. By opening the $100 \mathrm{~nm} \mathrm{Si}_{3} \mathrm{~N}_{4}$ passivation layers deposited on the active region by plasma-enhanced chemical vapor deposition (PECVD), the gate area of a $2 \mu \mathrm{m}$ gate length was defined. The $\mathrm{ZnO}$ NRs were then selectively grown as an active element for the UV light detection.
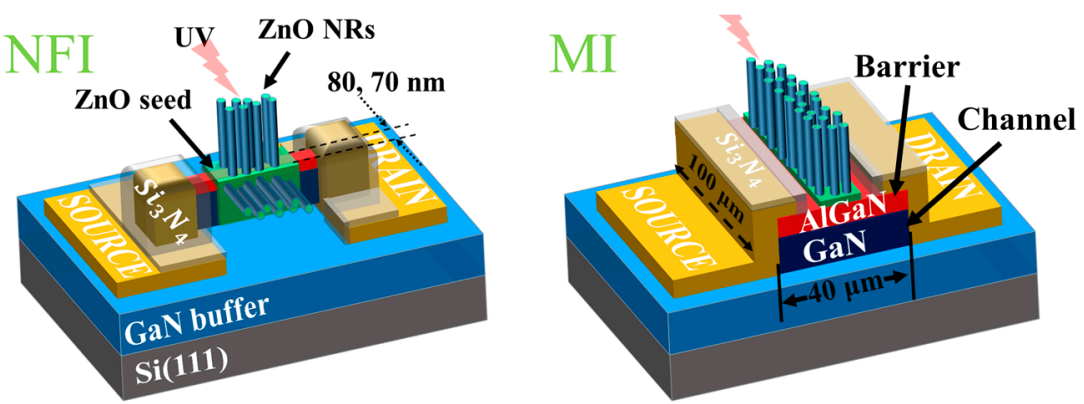

Figure 1. Schematics of the NFI (nanoscale fin isolation) and MI (mesa isolation) structures for the AlGaN/GaN HEMT (high electron mobility transistor)-based UV photodetectors.

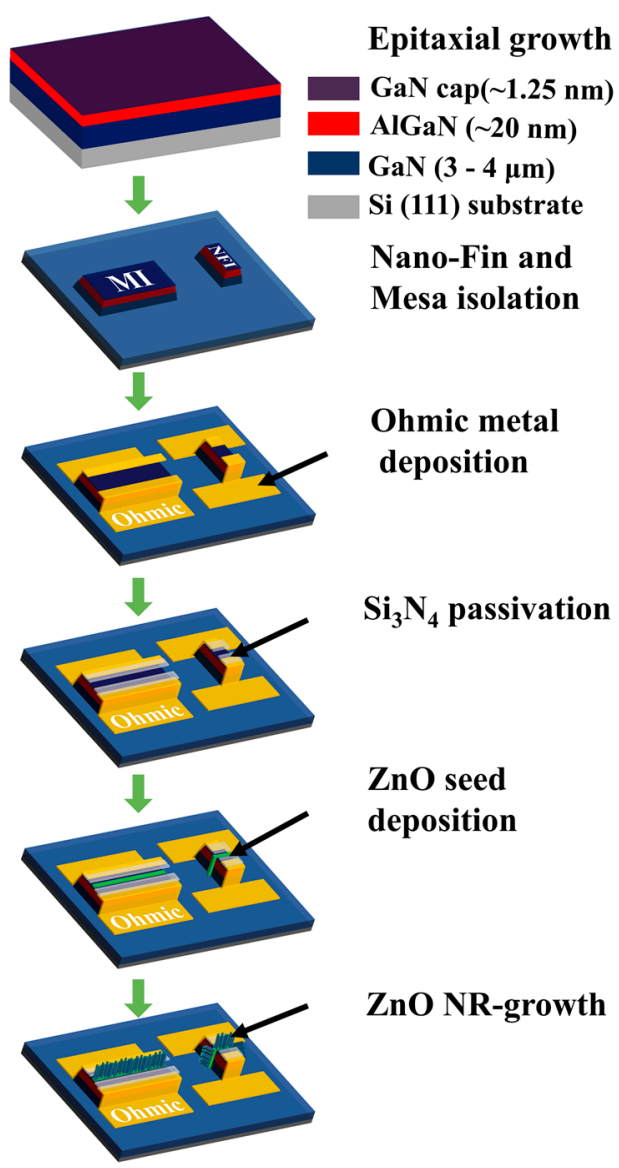

Figure 2. Schematic illustration of the major process steps used for the fabrication of the NFI and MI devices.

The epitaxial layers were cleaned and agitated with acetone and ethanol in bath sonication to remove dust and surface contamination, followed by rinsing in deionized (DI) water, then drying with nitrogen $\left(\mathrm{N}_{2}\right)$ gas. Optical lithography (Karl Suss, Garching, Germany, MA6 mask aligner, $365 \mathrm{~nm}$ ) was used to define the active MI regions, including all the device patterns used in this experiment. However, patterning for the NFIs was performed by an electron beam lithography tool (Jeol, Tokyo, 
Japan, JBX-9300FS, $100 \mathrm{keV}$ ) with a $70 \mathrm{~nm} \mathrm{PECVD} \mathrm{SiO}_{2}$ hard mask to avoid the mask pattern erosion during the mesa etching. After the pattern development of the active regions, mesa etching was performed by removing $100 \mathrm{~nm}$ of thickness from the peripheral areas in a reactive ion etching system (RIE, STS Multiplex ICP) using $\mathrm{BCl}_{3}$ and $\mathrm{Cl}_{2}$ gases. Ohmic contacts were achieved by depositing the metal stack of $\mathrm{Ti} / \mathrm{Ni} / \mathrm{Au}(20 / 30 / 80 \mathrm{~nm})$, by using an electron beam evaporation system, and a pattern lift-off method, using image reversal photoresist. Ohmic metals were then subjected to a subsequent rapid thermal alloy process at $900{ }^{\circ} \mathrm{C}$ for $35 \mathrm{~s}$ in $\mathrm{N}_{2}$. The samples were then passivated by a $100 \mathrm{~nm}$ silicon nitride $\left(\mathrm{Si}_{3} \mathrm{~N}_{4}\right)$ layer deposited in a PECVD system at $200{ }^{\circ} \mathrm{C}$ and RF power of $1 \mathrm{~kW}$ using a $\mathrm{NH}_{3} / \mathrm{SiH}_{4}$ gas flow rate ratio of 1.5. The gate areas $(2 \times 100 \mu \mathrm{m})$ of two different structures were opened by etching the $\mathrm{Si}_{3} \mathrm{~N}_{4}$ passivation using RIE in $\mathrm{CF}_{4}$ plasma at a gas flow rate of $110 \mathrm{sccm}$ and a chamber pressure of $40 \mathrm{mTorr}$ (at an etching rate of $\sim 9.4 \mathrm{~nm} / \mathrm{min}$ ) under the RF power of $100 \mathrm{~W}$.

$\mathrm{ZnO} N R s$ were then grown in the gate area by using the hydrothermal synthesis method. Prior to the growth of NRs, a $20 \mathrm{~nm}$ thick seed layer (SL) (as shown in the top-right inset of Figure 3c) was deposited by spin coating the seed solution ( $3000 \mathrm{rpm}$ and prebaking at $120^{\circ} \mathrm{C}$ for $60 \mathrm{~s}$ ) repeatedly 15 times. After that, the crystalline quality of the SL was improved by annealing the samples at $\sim 350{ }^{\circ} \mathrm{C}$ on a hotplate for $1 \mathrm{~h}$. The seed solution used in this study was prepared by dispersing $0.66 \mathrm{~g}$ of zinc acetate-dehydrate $\left(\mathrm{C}_{4} \mathrm{H}_{6} \mathrm{O}_{4} \mathrm{Zn} \cdot 2 \mathrm{H}_{2} \mathrm{O}\right)$ salt in $30 \mathrm{~mL}$ of 1-propanol $\left(\mathrm{CH}_{3} \mathrm{CH}_{2} \mathrm{CH}_{2} \mathrm{OH}\right)$. In this work, a $20 \mathrm{mM}$ zinc-acetate-dehydrate concentration for seed solution was selected for the desired SL film quality, assuring the ZnO NR crystalline characteristics to be used for this PD application [23]. Various attempts, such as vacuum annealing and $\mathrm{O}_{2}$ plasma post-treatment methods $[10,24]$ to improve the SL film quality, which is the key to the high-quality NR crystallites, are also underway in our laboratory. The growth of $\mathrm{ZnO}$ NRs was limited to the gate area, which was done by etching the SL from all other areas except the gate region. The samples were then placed in a growth solution for $6 \mathrm{~h}$ on a hotplate at $\sim 90{ }^{\circ} \mathrm{C}$ to grow $\mathrm{ZnO}$ NRs. The growth solution was prepared by mixing 0.25 mole equimolar concentration of zinc-nitrate-hexahydrate $\left(\mathrm{Zn}\left(\mathrm{NO}_{3}\right)_{2} \cdot 6 \mathrm{H}_{2} \mathrm{O}, 99 \%\right)$ and hexamethylenetetramine (HMTA) $\left(\mathrm{C}_{6} \mathrm{H}_{12} \mathrm{~N}_{4}, 99.5 \%\right)$ in deionized (DI) water. After growth of the the NRs, the samples were carefully cleaned with acetone, ethanol, and DI water, sequentially.

Sloped etching profiles for the NFI structure, the growth morphology of NRs, and the processed device structures were examined by plane-view scanning electron microscopy (SEM, $10 \mathrm{kV}$ S-4800S-Hitachi, Tokyo, Japan). As shown in the top-right inset of Figure 3a, the measured bottom dimensions of the trapezoidal fins were $170 \mathrm{~nm}$ after RIE for the $\boldsymbol{W}_{\text {fin }}$ of $70 \mathrm{~nm}$. The transmission electron microscope (TEM, 9500-Hitachi) was used to characterize the cross-sectional view and crystalline quality of the as-grown NRs. The RT photo-luminescence (PL) emission spectra from the as grown NRs was obtained by using a He-Cd laser illumination source with a $325 \mathrm{~nm}$ wavelength. Gate areas opened with a trench-shaped pattern inside the dark-shaded $\mathrm{Si}_{3} \mathrm{~N}_{4}$ passivation and the NRs grown on them are shown in the plane-view SEM micrographs in Figure 3a,b. An inset (bottom-right) of Figure 3a shows the morphology of the ZnO NRs grown around the underlying nano-fins in the gate area of the NFI structures at a magnification of $\times 3000$. Across the gate region, 10 fingers of NFIs are running in parallel, whereas the NRs are grown all the way vertically along the gate area in the MI structure, as shown in Figure $3 b$. The SEM image in Figure $3 c$ shows that the NRs exhibit an average diameter of $\sim 85 \mathrm{~nm}$ and a length of $\sim 1.4 \mu \mathrm{m}$. Figure $3 \mathrm{~d}$ shows a PL spectrum measured at RT from the as-grown $\mathrm{ZnO}$ NRs employed as a light absorbing structure in this work. PL characterization at RT is one of the most efficient tools to evaluate the crystalline quality for the WBG ZnO NRs of a direct band gap property. The spectrum exhibits a strong near band edge emission peak at a wavelength of $\sim 380 \mathrm{~nm}$, which is mainly associated with the band-to-band excitonic recombination of $\mathrm{ZnO}$ [25]. The near band edge emission intensity was increased about two times with the increase of the NR aspect ratio (AR, length/diameter) from $\sim 8$ to $\sim 16$. For this reason, NRs with an AR of $\sim 16$ were used in this experiment. Emissions in a visible range $(420-650 \mathrm{~nm})$ are due to various form of intrinsic defects, such as oxygen vacancy, zinc vacancy, and hydrogen and oxygen interstitials [10]. Despite the inevitable intrinsic defects and consequent visible emissions, as observed from most of 
the nano-crystallites grown through the aqueous solution based growth methods, our PL spectrum reveals fairly good optical properties in our $\mathrm{ZnO}$ crystallites compared to those of the NRs grown using similar methods $[2,26,27]$.
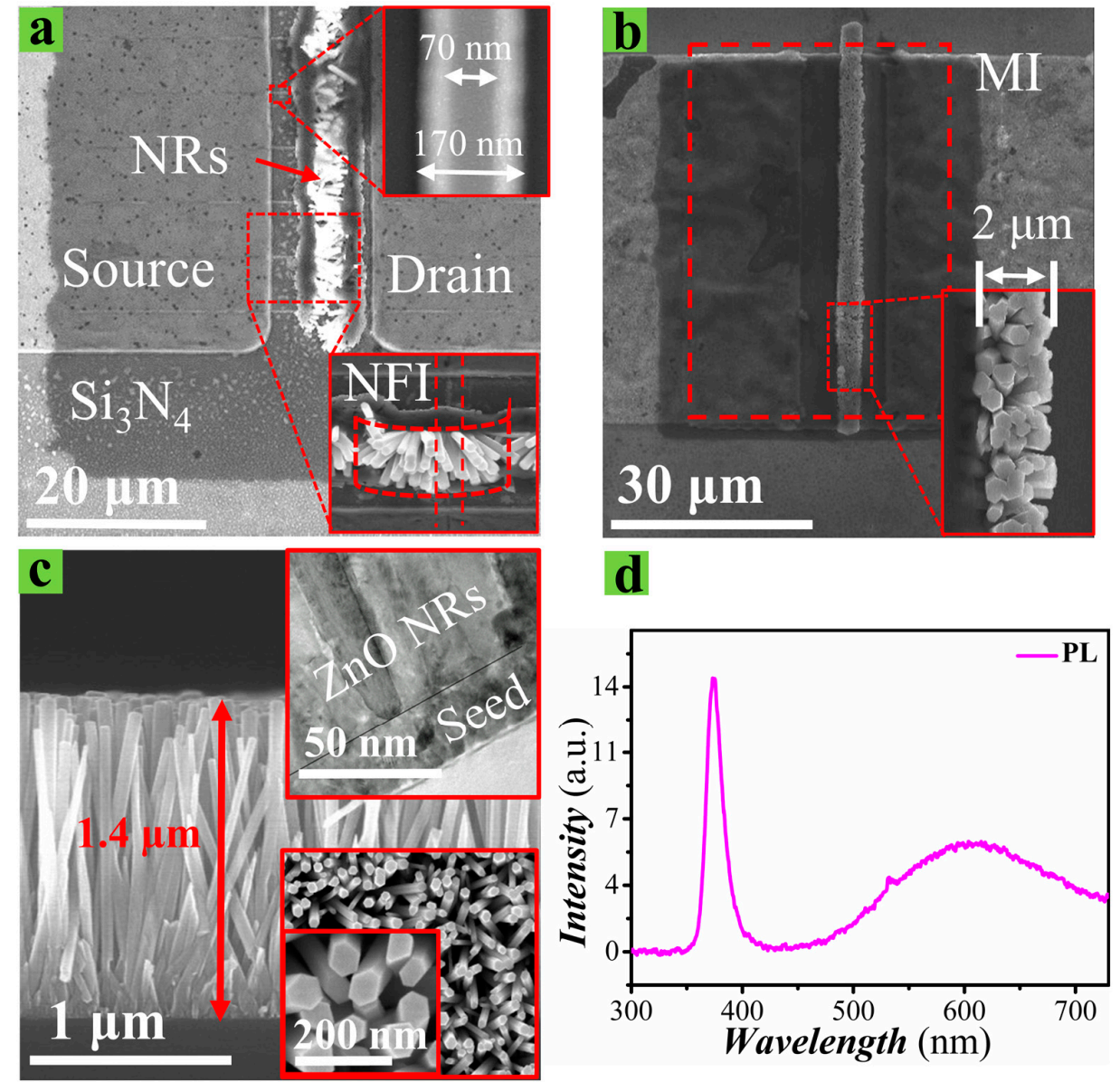

Figure 3. Plane-view SEM images of (a) $70 \mathrm{~nm}$ NFI PDs (photodetectors) $(\times 1000)$ with an enlarged top-view of the NFI $\left(\boldsymbol{W}_{f i n}=70 \mathrm{~nm}\right)$ (top-right inset, $\left.\times 5000\right)$ and $\mathrm{ZnO} \mathrm{NR}$ (nanorod) profiles (bottom-right inset, $\times 3000)$ and $($ b) MI PDs $(\times 500)$ with enlarged ZnO NRs profile (bottom-right inset, $\times 3000$ ). (c) Cross-sectional SEM image of the as-grown NRs. Cross-sectional TEM image of the NRs and SL (seed layer) (top-right inset). Top-view of the NRs (bottom-right inset). (d) RT (room temperature) PL (photo-luminescence) spectrum of the as-grown $\mathrm{ZnO}$ NRs.

A wide band (300-700 nm) Xenon (Xe) lamp was employed as a light source to measure the photoresponse characteristics of the fabricated devices under optical intensities ranging from 0.5 to $16.5 \mu \mathrm{W} / \mathrm{cm}^{2}$ (100-300 W lamp power). The transient characteristic measurements, according to the UV light $(370 \mathrm{~nm})$ on-and-off transient, were carried out by a programmable light shutter controlled in our measurement set-up. The transients of the drain current $\left(\boldsymbol{I}_{d s}\right)$ for the HEMT PDs as a function of time were recorded in a Keithley source measurement unit with a floating gate configuration at a drain voltage $\left(V_{d s}\right)$ of $4 \mathrm{~V}$. The spectral response of the PDs was measured by a focused illumination of a monochromatic light from a wide band (300-1100 nm) Xe-lamp (Ushio UXL-75XE, Ushio Inc., Tokyo, Japan) light source of a $16.5 \mu \mathrm{W}$ incident optical power. In this measurement set-up, the responsivity was measured at a light chopping frequency of $30 \mathrm{~Hz}$ using a lock-in amplifier in a series configuration with drain-source probes for the detection of an amplified change in current. The change of $\boldsymbol{I}_{\boldsymbol{d} s}$ as a function of incident light wavelength varying from 300 to $800 \mathrm{~nm}$ was recorded under a floating gate configuration at a $V_{d s}$ of $4 \mathrm{~V}$. To obtain a monochromatic light from the Xe-lamp, a spectral optics monochromator (CM110 1/8 m) with 2400 lines/mm grating was used. 


\section{Sensing Mechanism}

As illustrated in the schematics of Figure 4, the UV sensing mechanism of the HEMT based PDs depends on the chemisorption of oxygen at the surface of NRs and the consequent change in carrier concentration in underlying 2-DEG. The as-grown crystalline $\mathrm{ZnO}-\mathrm{NRs}$ are n-type in nature due to a large number of donor defects, such as hydrogen interstitial and $\mathrm{Zn}$ interstitial [28]. Under dark conditions (no UV light illumination), oxygen molecules $\left(\mathrm{O}_{2}\right)$ transported from the ambient air to the $\mathrm{ZnO} N R$ surface tend to trap electrons from the conduction band of $\mathrm{ZnO}$ and leave behind positively charged ionized donors in the surface space charge region, while the negatively charged $\mathrm{O}_{2}$ molecules are fixed to the surface of NRs as adsorbed oxygen ions $\left(\mathrm{O}_{2_{\text {ads }}^{-}}^{-}\right)$:

$$
\mathrm{O}_{2}+e^{-} \rightarrow \mathrm{O}_{2 \text { ads }}^{-} \text {. }
$$

This reaction leads to the expansion of the space charge region near the surface of the NRs due to the depletion of the surface electron states by $\mathrm{O}_{2_{\text {ads }}}^{-}$as depicted in the left of Figure 4a. As a consequence, the adsorption process gives rise to a negative potential at the gate of our HEMT based PDs (as shown in the left of Figure $4 b, c$ ), thereby dropping the carrier concentration in the 2-DEG channel at the interface of $\mathrm{AlGaN} / \mathrm{GaN}$. This process will eventually reduce the conductance of channel and drain-to-source current $\left(\boldsymbol{I}_{\boldsymbol{d s}}\right)$ in dark conditions. The Schottky gate (Ni/Au) HEMTs $(2 \mu \mathrm{m}$ gate length, $100 \mu \mathrm{m}$ gate width) fabricated in our group using the same epitaxial structure and $\mathrm{Si}_{3} \mathrm{~N}_{4}$ passivation showed a threshold voltage $\left(\boldsymbol{V}_{T H}\right)$ of $\sim-3 \mathrm{~V}$ [29]. This result demonstrates that our HEMT devices with an $\mathrm{Au} / \mathrm{Ni}$ gate electrode are normally on at zero gate voltage $V_{g s}$ (depletion-mode). We suppose that the $V_{T H}$ of the HEMTs is given by [30]

$$
\varnothing_{b}-\Delta E_{c}-\left(e n_{s} d^{2}\right) / 2 \varepsilon
$$

where $\varnothing_{b}$ is the Schottky barrier height, $\Delta E_{c}$ is the conduction band offset, $e$ is the electron charge, $n_{s}$ is the 2-DEG sheet carrier density, $\boldsymbol{d}$ is the barrier layer thickness, and $\varepsilon$ is the dielectric constant. Because the difference between the estimated $\varnothing_{b}$ of $\mathrm{Ni}$ at the interface with $\mathrm{GaN}(\sim 1.1 \mathrm{eV})$ [31] and the GaN surface band bending ( 1.0 eV) [32] in ambient air (fully saturated by $\mathrm{O}_{2_{\text {ads }}^{-}}^{-}$in the dark state) is quite small, it can be reasonably assumed that a significant amount of dark current $\left(I_{\text {dark }}\right)$ through the 2-DEG channel is unavoidable from our gateless MI HEMT devices under a floating gate condition due to their normally-on characteristics as shown in Figure 4c.

On the other hand, a different mode of operation is expected for the NFI structure because the 2-DEG channel region inside the nano-fins is three-dimensionally surrounded by a free surface, whereon the $\mathrm{ZnO}$ NRs are grown with many $\mathrm{O}_{2 \text { ads }}^{-}$around the surface in dark conditions, as shown in Figure $4 \mathrm{~b}$. This makes the NFI PDs operate more closely to a normally-off mode (enhancement mode), thereby exhibiting a much lower $\boldsymbol{I}_{\text {dark }}$, because the carriers in the 2-DEG region can be highly depleted, even under the floating gate conditions, due to the surface depletion of the NRs by the $\mathrm{O}_{2_{\text {ads }}^{-}}^{-}$surrounding the extremely small volume of the active region. The same phenomenon was also observed in the AlGaN/GaN heterojunction FinFETs [33]. With the decrease of $\boldsymbol{W}_{\text {fin }}$ from 200 to $60 \mathrm{~nm}$, it was observed from the HEMT devices that electron density in the 2-DEG channel at zero $V_{g s}$ rapidly drops with a positive shift in $V_{T H}$ because of the fringing-field from the side gates depleting the 2-DEG channel. As a result, the fin-HEMT showed a change in the conduction mechanism from normally-on to normally-off modes.

Due to light absorption, UV light illumination generates electron-hole pairs near the surface of the $\mathrm{ZnO}$ NRs [34]. The generated holes recombine with the electrons trapped by $\mathrm{O}_{2_{\text {ads }}^{-}}$at the surface. In this way, the $\mathrm{O}_{2}$ molecules start to desorb from the surface, as shown in the right of Figure 4a. This phenomenon gives rise to a reduction of negative charge in the gate region, thereby increasing the carrier concentration in the 2-DEG channel and the drain to the source current $\left(\boldsymbol{I}_{\text {photo }}\right)$ under UV illumination, as shown in the right parts of Figure $4 b, c$. 


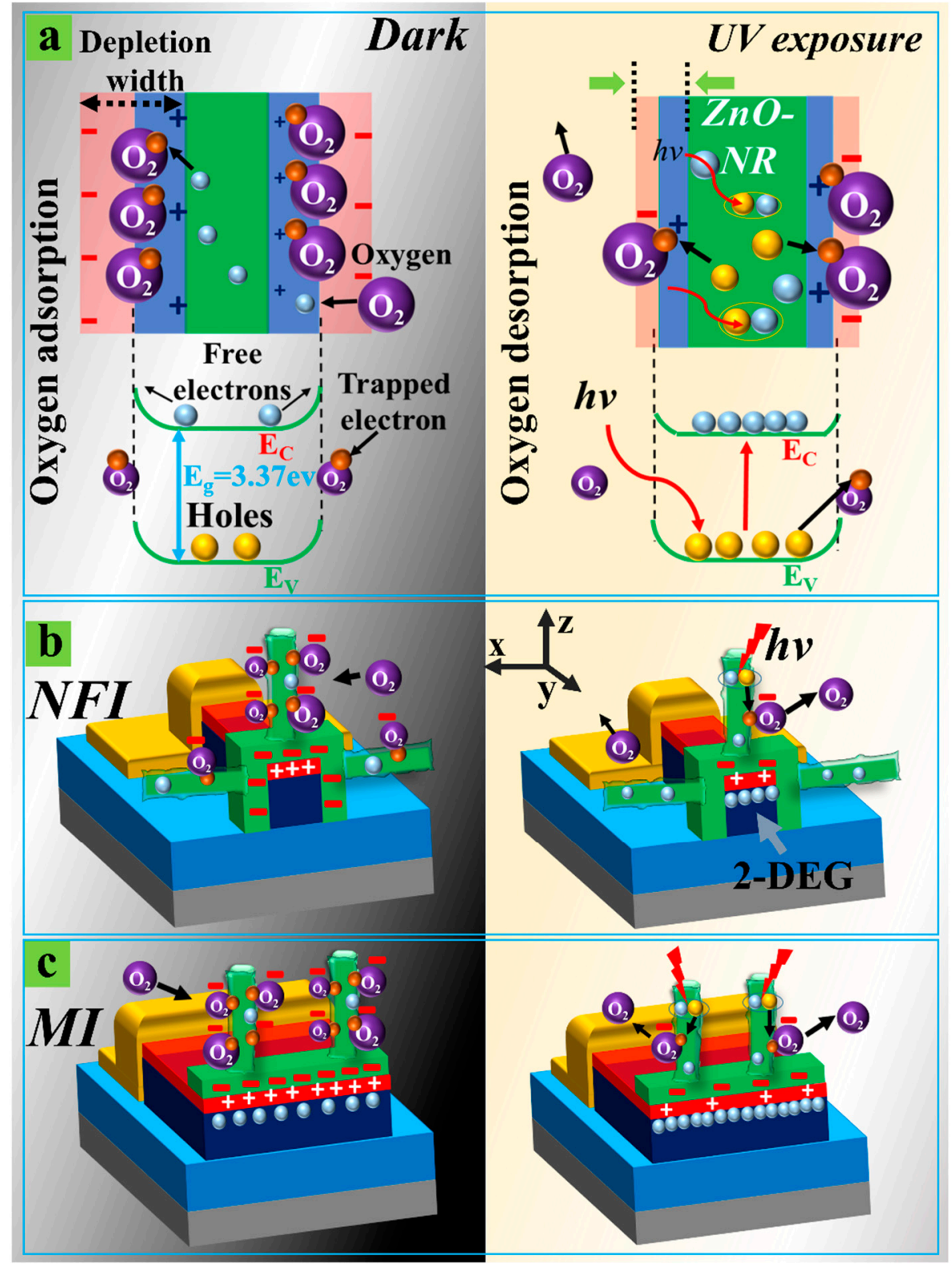

Figure 4. Schematic illustrations of (a) oxygen adsorption (left: in the dark) and desorption (right: under UV illumination) at the surface of ZnO-NRs. Oxygen adsorption (left: in the dark) and desorption (right: under UV illumination) processes are taking place at the surface of ZnO NRs grown on the gate areas of (b) NFI and (c) MI PDs. Cross-sectional schematics of each PD structure viewed in y-axis direction are illustrated; 2-Deg (two-dimensional electron gas).

As long as the response speed of PDs is accounted for, it can be assumed that the response kinetics upon the UV light on-and-off transient are not controlled by the drift motion of electrons in the 2-DEG channel but by the adsorption and desorption reaction of $\mathrm{O}_{2}$ on the $\mathrm{ZnO}$ NR surface. This result occurs because the mobility of the electrons confined two-dimensionally in the channel region $\left(\sim 1750 \mathrm{~cm}^{2} \mathrm{~V}^{-1} \mathrm{~s}^{-1}\right)[33,35]$ is so fast that the carrier channel transit time cannot be a bottleneck parameter controlling the whole sensor response speed. As proposed in our previous study, the rate of charge change $d Q / d t$ can be given by [35]:

$$
d Q / d t=-\alpha e^{\beta Q}
$$


where $\alpha$ and $\beta$ are the constants. By numerically solving Equation (3), it can be determined that one critical parameter affecting the response time is the gate area. From the calculations based on this model, the response (or recovery) time increases with the increase of gate area caused by the consequent increase of the total gate charge. We have much smaller gate capacitance in the NFI PD structure than in the MI structure; therefore, a faster response speed upon UV transient illumination can be expected from the NFI structure.

Spectral responsivity $\boldsymbol{R}$, which can be defined as a ratio of $\boldsymbol{I}_{\text {photo }}-\boldsymbol{I}_{\text {dark }}$ to the incident optical power $\boldsymbol{P}_{\boldsymbol{i}}$, is one of the key measures to evaluate the performance of PDs. As reported in our previous studies [19,35], a significant enhancement in $\boldsymbol{R}$ was shown from the NR-gated PDs due to a vast surface area of the $\mathrm{ZnO}$ nanostructure and a much higher surface-to-volume ratio than that of the planar $\mathrm{ZnO}$ thin film gate structure. In our HEMT-based PDs, the $\boldsymbol{R}$ can be also significantly influenced by the gain characteristic for the HEMT, which can be expressed by the change of $\boldsymbol{I}_{\text {photo }}$ according to the change of light power irradiating on the gate area.

\section{Results and Discussion}

Figure 5a shows the equivalent electrical circuit diagram of both MI and NFI devices, where MI PD is illustrated as a normally-on transistor while the NFI PD is a normally-off transistor. To examine the characteristic difference of UV responses from the two different PD structures, we first measured $I_{d s}$ in dark conditions $\left(\boldsymbol{I}_{d a r k}\right)$ and under UV illumination $\left(\boldsymbol{I}_{\text {photo }}\right)$ at drain voltage $\left(\boldsymbol{V}_{\boldsymbol{d s}}\right)$, ranging from 0-5 V. UV light exposure was provided from an Xe lamp, operating at $300 \mathrm{~W}$ power, with a monochromatic light filter at $370 \mathrm{~nm}$. This UV source produced an incident light intensity of $16 \mu \mathrm{W} / \mathrm{cm}^{2}$, as measured by a power meter. Figure $5 \mathrm{~b}$ demonstrates that our MI PD exhibits a high $\boldsymbol{I}_{\text {dark }}$ of $\sim 10 \mathrm{~mA} / \mathrm{mm}$ at a $V_{d s}$ of $4 \mathrm{~V}$ due to the enhancement-mode operation, as discussed in the previous section. In the case of NFI PDs, significantly reduced values of $\boldsymbol{I}_{\text {dark }}\left(\sim 0.19\right.$ and $\sim 0.27 \mathrm{~mA} / \mathrm{mm}$ for $\boldsymbol{W}_{\text {fin }}$ of 70 and $80 \mathrm{~nm}$, respectively) were measured at a $V_{d s}$ of $4 \mathrm{~V}$.
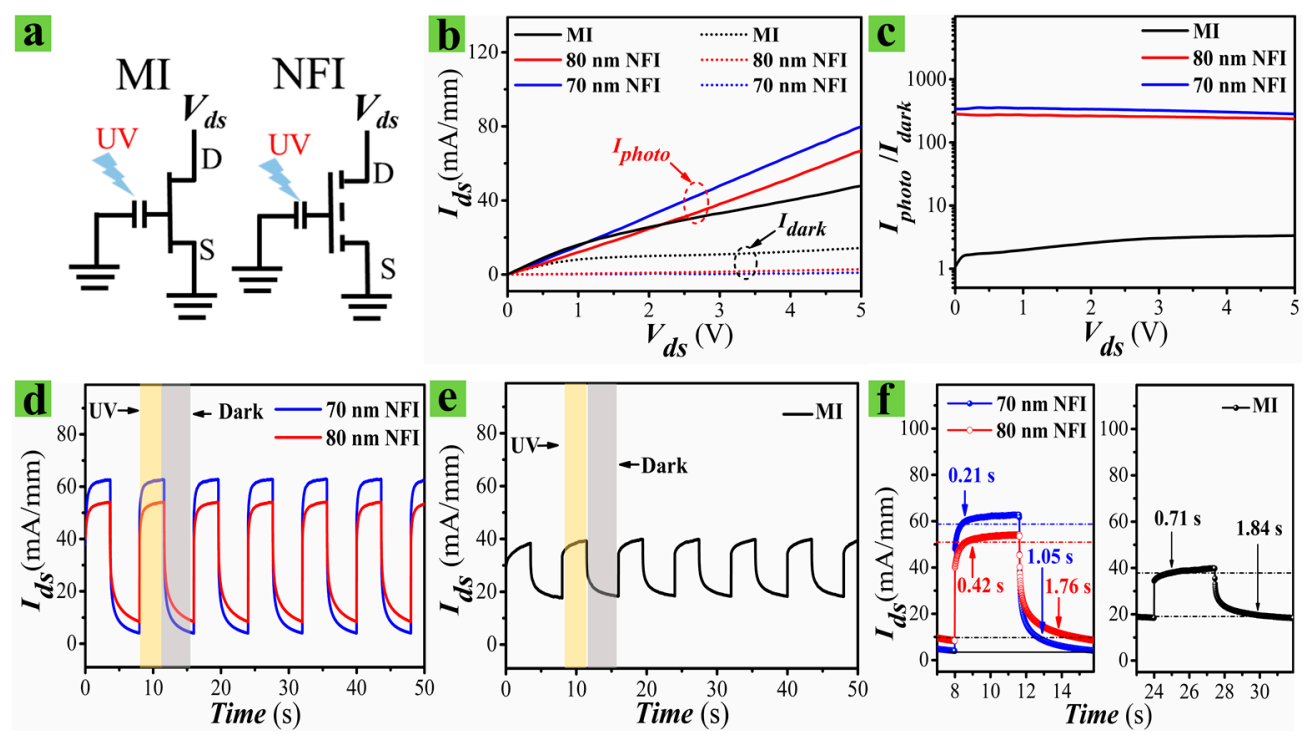

Figure 5. Photoresponse of MI and NFI PDs. (a) Equivalent electrical circuits of MI (left) and NFI devices (right). (b) Measured $I_{d s}$ versus $V_{d s}$ and (c) on-off current ratio $\left(I_{\text {photo }} / I_{\text {dark }}\right)$ of MI and NFI PDs. Time resolved photoresponse characteristics of (d) NFI and (e) MI PDs $\left(V_{d s}=4 \mathrm{~V}\right)$. (f) Magnified views of transient characteristics of NFI (left) and MI (right) PDs.

The performance of PDs can be evaluated by few important parameters, such as spectral responsivity $R$, photoconductive gain $G$, specific detectivity $D^{*}$, and sensitivity $S$ [36]. Despite the high $\boldsymbol{R}$ nature of our MI HEMT-based PDs, this high $\boldsymbol{I}_{\text {dark }}$ of the MI structure can critically deteriorate the photo-sensitivity performance associated with $S$, which is given by $\left.\left(\boldsymbol{I}_{\text {photo }}-\boldsymbol{I}_{\text {dark }}\right) / \boldsymbol{I}_{\text {dark }}\right)$, or the on-off 
current ratio $\left(\boldsymbol{I}_{\text {photo }} / \boldsymbol{I}_{\text {dark }}\right)$, as well as $\boldsymbol{D}^{*}$. As shown in Figure $5 \mathrm{c}$, much improved on-off current ratios (290 340) were recorded from the NFI PDs compared to those of the MI devices ( 4). This result is mainly due to the suppression of $\boldsymbol{I}_{\text {dark }}$ caused by the fully depleted 2-DEG channel of the NFI devices in dark conditions.

The high-speed transient characteristic of the UV PD is one of the key factors for real-time application. Specific cases, such as the non-invasive assessment of cancer cells by optical biopsy [37], require ultra-fast PDs with response and recovery time in the order of a few milliseconds. To assess the photocurrent transient of the fabricated devices, the change in $I_{d s}$ as a function of the on-and-off of the UV light exposure time was measured using a programmable shutter. Figure $5 \mathrm{~d}$,e shows that both the NFI and MI PDs produce sharp increases in $I_{d s}$ upon UV illumination and a slower fall-off upon termination of UV exposure. The NFI device with a $70 \mathrm{~nm} \boldsymbol{W}_{\text {fin }}$ showed the fastest rise-up time (or UV response time) and fall-off time (or recovery time) of 0.21 and $1.05 \mathrm{~s}$, respectively. We hereafter define the rise-up and fall-off time as the time intervals for $\boldsymbol{I}_{\boldsymbol{d}}$ to ramp up to $90 \%$ of the maximum saturation value after UV turn-on and to ramp down by $90 \%$ from the maximum value after UV-off. On the other hand, the MI device showed much slower response and recovery times of $\sim 0.71 \mathrm{~s}$ and $\sim 1.84 \mathrm{~s}$, respectively. This significant improvement in response speed of the NFI device is due to the minimized dimension of gate area where the light absorption takes place; therefore, less time is required to complete the $\mathrm{O}_{2}$ adsorption-desorption process in the smaller area of the NFI PDs than in the larger area of the MI device, as discussed in the previous section. The $\mathrm{ZnO} \mathrm{NR}$ based photoconductive PDs [26,38] still show a long recovery time on a seconds scale, even though a great amount of research effort has been made on enhancing the response speed of these passive PDs. UV PDs based on $\mathrm{ZnO}$ nanowire networks with Pt contacts have been fabricated on glass substrates by exhibiting a fast recovery time of $0.2 \mathrm{~s}$ with a high photosensitivity $\left(\sim 5 \times 10^{3}\right)$ at $365 \mathrm{~nm}$ [39]. The fastest UV PDs of GaN-based metal-semiconductor-metal, p-i-n, or metal Schottky barrier devices [6] have shown extremely high speed (from microseconds to picoseconds) and low-noise capabilities. However, PDs of these GaN-based structures, developed specially to improve the UV response characteristics, exhibit a very low spectral responsivity of less than $1 \mathrm{~A} / \mathrm{W}$ [8].

Figure 6a shows the schematic illustration of PD before and after the growth of the ZnO NRs. Shown in Figure $6 \mathrm{~b}, \mathrm{c}$ are the measured $\boldsymbol{I}_{\text {dark }}$ values of the two different device structures before and after NR growths on the gate region. For the MI PDs, the dark currents were significantly reduced by the attachment of NRs on the gate region. However, the $\boldsymbol{I}_{\text {dark }}$ values measured over the entire drain voltage range were still very high $(\sim 10 \mathrm{~mA} / \mathrm{mm})$ due to the incomplete depletion of the 2-DEG channel in a large volume of the active region. The measured $\boldsymbol{I}_{\text {dark }}$ of the NFI PDs before the NR growth were very high, reaching up to $\sim 560 \mathrm{~mA} / \mathrm{mm}$, but they rapidly dropped to a very low level of $\sim$ hundreds of $\mu \mathrm{A} / \mathrm{mm}$ by the attachment of NRs. This result reveals that both the surface depletion effect in the small volume of an active region and the attachment of the NRs with many $\mathrm{O}_{2}^{-}$ads on their surfaces lead to the formation of a fully-depleted 2-DEG channel in the NFI structure.
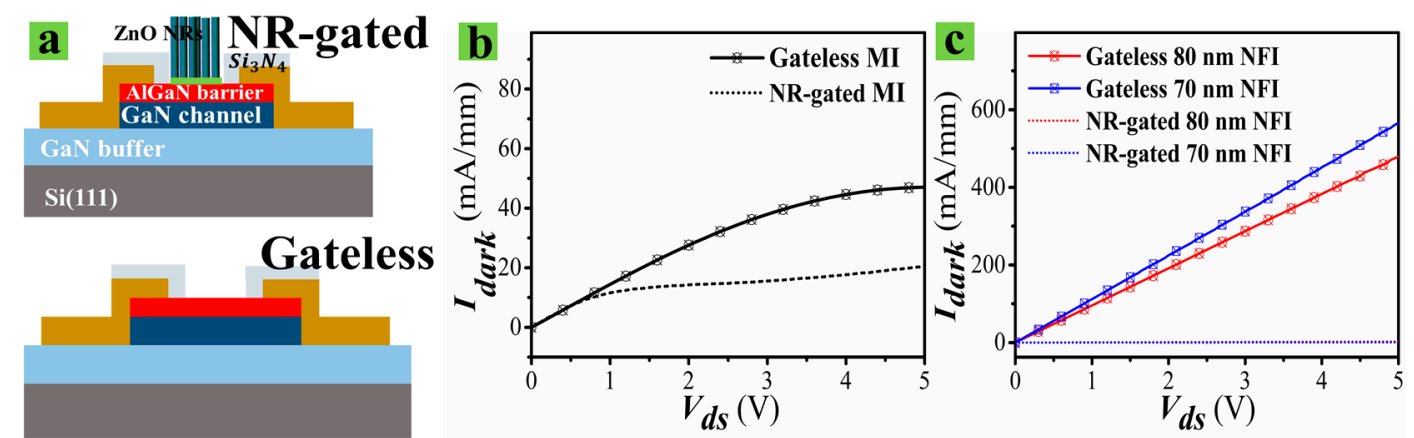

Figure 6. (a) Schematic illustration of NR-gated PD and Gateless PD. Measured $\boldsymbol{I}_{\text {dark }}$ of MI (b) and NFI (c) device structure before (gateless) and after NR growths (NR-gated). 
The performance of the PDs can be also assessed by spectral responsivity $R$ and specific detectivity $D^{*}$, which are expressed in the following equations [36]:

$$
\begin{gathered}
R=\frac{I_{\text {photo }}-I_{\text {dark }}}{P_{i}} \\
D *=\sqrt{A} R / \sqrt{2 q I_{\text {dark }}}
\end{gathered}
$$

where $\boldsymbol{A}$ is the active area of device, $\boldsymbol{q}$ is the electron charge, and $\boldsymbol{P}_{\boldsymbol{i}}$ is the radiant light power incident on the active area of the device. The responsivities of our PDs were measured using an incident light intensity of $16 \mu \mathrm{W} / \mathrm{cm}^{2}$ with an effective area of $2.4 \times 2.4 \mathrm{~mm}^{2}$. NFI PDs showed an extremely high responsivity of $\sim 3.2 \times 10^{7} \mathrm{~A} / \mathrm{W}$ at $340 \mathrm{~nm}$, which is the best performance among any solid-state PDs reported to date [40-44], and even $\sim 100$ times higher than that $\left(\sim 2 \times 10^{5} \mathrm{~A} / \mathrm{W}\right.$ at $\left.340 \mathrm{~nm}\right)$ of the MI device, as shown in the right of Figure 7a. This improvement in responsivity of the NFI PD is attributed to a higher photonic transconductance (hereafter, we call it $g_{m, p h o t o}$ ) characteristic of our HEMT-based PDs, which represents the ratio of the photocurrent change at the drain terminal to the change in incident optical power at the gate terminal. We define the $g_{m, p h o t o}$ as following.

$$
g_{m, \text { photo }}=\frac{\Delta \boldsymbol{I}_{\text {photo }}}{\Delta \boldsymbol{P}_{i}}(A / W)
$$
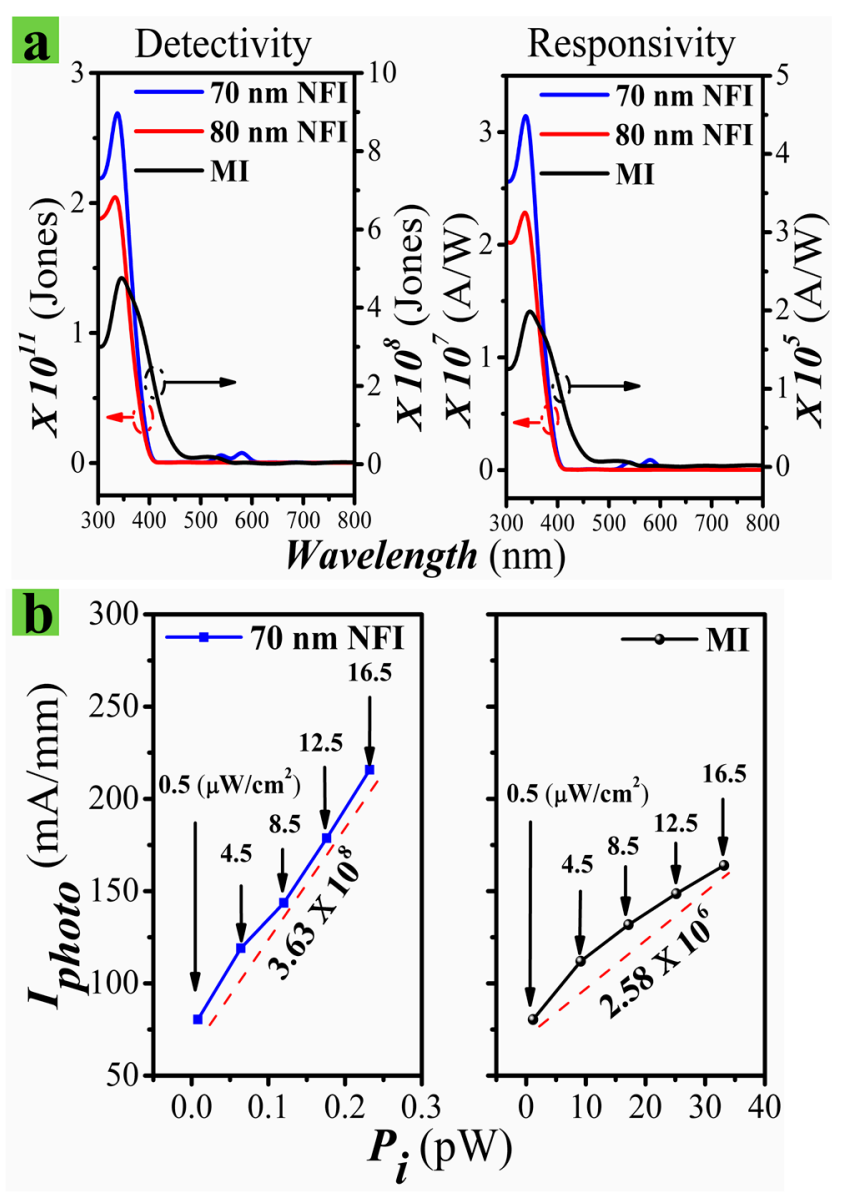

Figure 7. (a) Specific detectivity (left) and spectral responsivity (right) as functions of radiant light wavelength measured from NFI and MI UV detectors. (b) $\boldsymbol{I}_{\text {photo }}$ versus $\boldsymbol{P}_{\boldsymbol{i}}$ of the NFI (left) and MI (right) PDs measured at $V_{d s}$ of $5 \mathrm{~V}$. 
To calculate the $g_{m, \text { photo }}$ values of the two different device structures, the $\boldsymbol{I}_{\text {photo }}$ values were measured at various optical intensities, varying from 0.5 to $16 \mu \mathrm{W} / \mathrm{cm}^{2}$ at a $V_{d s}$ of $5 \mathrm{~V}$. Shown in Figure $7 \mathrm{~b}$ is the measured $\boldsymbol{I}_{\text {photo }}$ versus $\boldsymbol{P}_{\boldsymbol{i}}$ from the MI and NFI PDs. The estimated $\boldsymbol{g}_{m, \text { photo }}$ values for the NFI and MI PDs were $3.63 \times 10^{8}$ and $2.58 \times 10^{6} \mathrm{~A} / \mathrm{W}$, respectively. Even though this $g_{m, \text { photo }}$ parameter does not directly represent the gain $\left(g_{m}=\Delta I_{d s} / \Delta V_{g s}\right)$ characteristics of the field-effect transistors, it can be used as a performance measure to estimate the gain characteristic of our gateless HEMT PDs, because the change of incident optical power is directly associated with the virtual $\Delta V_{g s}$ induced by the change in numbers of $\mathrm{O}_{2}^{-}$ads on the $\mathrm{ZnO}$ NR surface under the illumination of UV light.

FinFET technology has recently shown a major increase in adoption of use within Si integrated circuits. The advantages of a FINFET structure, even though there are a number of subtly different forms, can be numerous, but they are basically based on "channel controllability" in a nano-scale channel-length regime of FETs [45]. Furthermore, Si-based FINFETs have shown a significant enhancement in the dependence of $\boldsymbol{I}_{\boldsymbol{d s}}$ on $\boldsymbol{V}_{\boldsymbol{g}}$ at any applied bias in the sub- and near-threshold regimes by the superior electrostatics of the device architecture [46]. Normally-off $\mathrm{Al}_{2} \mathrm{O}_{3} / \mathrm{GaN}$ metal-insulator FINFETs $\left(W_{f i n}=50 \mathrm{~nm}, 1 \mu \mathrm{m}\right.$ gate length) also showed very high maximum $I_{d s}, g_{m}$, and maximum field-effect mobility of $1.51 \mathrm{~A} / \mathrm{mm}, 580 \mathrm{mS} / \mathrm{mm}$, and $293 \mathrm{~cm}^{2} \mathrm{~V}^{-1} \mathrm{~s}^{-1}$, respectively, due to the more effective increase of 2-DEG electron concentration and higher electron mobility by enhanced gate controllability than the planar devices [47,48]. The superior performance of $\boldsymbol{R}$ in our NFI PDs is most likely attributed to this enhancement of "gate controllability" screening the field lines effectively from the interface traps or the defects near the 2-DEG channel, thereby reducing the electron scattering in the GaN channel.

The $D^{*}$ of a PD, as defined in Equation (5), is also an important figure of merits used to describe performance. The NFI PDs of $70 \mathrm{~nm} \boldsymbol{W}_{\text {fin }}$ exhibited a maximum $\boldsymbol{D}^{*}$ of $\sim 3.2 \times 10^{12} \mathrm{Jones}\left(\mathrm{cm} \cdot \mathrm{Hz}^{-1 / 2} / \mathrm{W}\right)$ at $340 \mathrm{~nm}$, which is four orders of magnitude higher than that of MI PDs $\left(\sim 4 \times 10^{8}\right.$ Jones), as shown in the left of Figure 7a, which is mainly due to the very low $I_{\text {dark }}$ of the NFI structure. A very high $D^{*}$ up to a value of $1.4 \times 10^{15}$ Jones [14] was reported from the ZnO NRs based UV PDs. However, the $\boldsymbol{R}$ achieved by this device was much lower $\left(\sim 10^{3} \mathrm{~A} / \mathrm{W}\right)$ than that of PDs.

\section{Conclusions}

Most of the performance parameters, such as $R, D^{*}$, on-off current ratio, and response speed, were all significantly improved by employing the NFI structure for the AlGaN/GaN HEMT based UV PDs with a ZnO NR UV-absorbing structure. The NFI PD, especially, exhibited an extremely high $R$ of $\sim 3.2 \times 10^{7} \mathrm{~A} / \mathrm{W}$. This performance enhancement was due to the subsequent characteristic change of the gateless HEMTs, induced by the reduction of $\boldsymbol{W}_{f i n}$ to $70-80 \mathrm{~nm}$. The NFI structure significantly improved the gain characteristics caused by enhanced gate controllability in nano-fin channels beyond the inherent high performance in conversion efficiency of the photon to electron-hole pair generation due to the large surface-to-volume ratio of the $\mathrm{ZnO}$ NRs grown in the active region. As the width of the 2-DEG channel is reduced by the NFI profile, the side-wall surface depletion in nano-fins and the attachment of NRs with numerous $\mathrm{O}_{2 \text { ads }}^{-}$on the surface of the NRs lead to the formation of a fully-depleted 2-DEG channel and pushed the $V_{T H}$ to a positive value. The measured high on-off current ratio and $D^{*}$ are mainly due to this normally-off operation of the NFI PD structure. An improvement in response speed of the PDs is associated with the minimized dimension of the gate area and the resulting gate capacitance of the NFI structure, where much less time for charging or discharging is required for the $\mathrm{O}_{2}$ adsorption-desorption process. The fabricated PDs also showed a linear dependence of photocurrent on the input light intensity in a range of $0.5-16.5 \mu \mathrm{W} / \mathrm{cm}^{2}$, regardless of device structure. The measured $g_{m \text {,photo }}$ value for the NFI PDs of $70 \mathrm{~nm} \boldsymbol{W}_{f i n}$ was $3.63 \times 10^{8} \mathrm{~A} / \mathrm{W}$, which was $\sim 100$ times greater than that of the MI PDs.

Author Contributions: Conceptualization, F.K.; methodology, F.K.; validation, F.K.; formal analysis, F.K.; investigation, F.K.; resources, S.-D.K.; data curation, F.K.; writing-original draft preparation, F.K.; writing一review 
and editing, F.K. and S.-D.K.; visualization, S.-D.K.; supervision, S.-D.K. and W.K.; project administration, S.-D.K.; funding acquisition, S.-D.K.

Funding: All the experiments performed in this work are financially sponsored by National Research Foundation (NRF) grant (2016006533) under the program: "Mid-career Researchers" by the Ministry of Science and Technology, Republic of Korea. Similarly, the materials characterization was achieved with the financial support from NRF, Republic of Korea under the program: "Nano-materials Technology Development" (2009-0082580) managed by Ministry of Science, ICT and Future Planning.

Acknowledgments: The authors would like to thank Jihyun Kim, Hafiz Muhammad Salman Ajmal, Kiyun Nam and Noor ul Huda from Dongguk University for the continued encouragement, help, and support in carrying out the experiments and preparation of results.

Conflicts of Interest: The authors declare no conflicts of interest.

\section{References}

1. Bigio, I.J.; Mourant, J.R. Ultraviolet and visible spectroscopies for tissue diagnostics: Fluorescence spectroscopy and elastic-scattering spectroscopy. Phys. Med. Biol. 1997, 42, 803-814. [CrossRef] [PubMed]

2. Monroy, E.; Omns, F.; Calle, F. Wide-bandgap semiconductor ultraviolet photodetectors. Semicond. Sci. Technol. 2003, 18, R33-R51. [CrossRef]

3. $\mathrm{Li}, \mathrm{Z}$. Radiation damage effects in Si materials and detectors and rad-hard Si detectors for SLHC. J. Instrum. 2009, 4, P03011. [CrossRef]

4. Nanver, L.K.; Qi, L.; Mohammadi, V.; Mok, K.R.M.; Boer, W.B.D.; Golshani, N.; Sammak, A.; Scholtes, T.L.M.; Gottwald, A.; Kroth, U.; et al. Robust UV/VUV/EUV PureB Photodiode Detector. IEEE J. Sel. Top. Quantum Electron. 2014, 20, 306-316. [CrossRef]

5. Qi, L.; Mok, K.R.C.; Aminian, M.; Charbon, E.; Member, S.; Nanver, L.K. UV-sensitive low dark-count pureb single-photon avalanche diode. IEEE Trans. Electron Devices 2014, 61, 3768-3774. [CrossRef]

6. Omnès, F.; Monroy, E.; Muñoz, E.; Reverchon, J.-L. Wide bandgap UV photodetectors: A short review of devices and applications. In Gallium Nitride Materials and Devices; Integrated Optoelectronic Devices: San Jose, CA, USA, 2007; p. 64730E.

7. Falson, J.; Kozuka, Y.; Uchida, M.; Smet, J.H.; Arima, T.; Tsukazaki, A.; Kawasaki, M. MgZnO/ZnO heterostructures with electron mobility exceeding $1 \times 106 \mathrm{~cm}^{2} /$ Vs. Sci. Rep. 2016, 6, 26598. [CrossRef] [PubMed]

8. Al-Heuseen, K.; Hashim, M.R. Enhancing responsivity of porous GaN metal-semiconductor-metal ultraviolet photodiodes by using photoelectrochemical etching. Acta Phys. Pol. A 2012, 121, 71-73. [CrossRef]

9. Melnick, D.A. Zinc Oxide Photoconduction, an Oxygen Adsorption Process. J. Chem. Phys. 1957, 26, 1136-1146. [CrossRef]

10. Khan, W.; Khan, F.; Ajmal, H.; Huda, N.; Kim, J.; Kim, S.-D. Evolution of structural and optical properties of zno nanorods grown on vacuum annealed seed crystallites. Nanomaterials 2018, 8, 68. [CrossRef] [PubMed]

11. Humayun, Q.; Kashif, M.; Hashim, U.; Qurashi, A. Selective growth of ZnO nanorods on microgap electrodes and their applications in UV sensors. Nanoscale Res. Lett. 2014, 9, 29. [CrossRef] [PubMed]

12. Mohammad, S.M.; Hassan, Z.; Ahmed, N.M.; Al-Hardan, N.H.; Bououdina, M. Fabrication of low cost UV photo detector using $\mathrm{ZnO}$ nanorods grown onto nylon substrate. J. Mater. Sci. Mater. Electron. 2015, 26, 1322-1331. [CrossRef]

13. Khan, W.; Kim, S.-D. Ultra-violet photo-response characteristics of $\mathrm{p}-\mathrm{Si} / \mathrm{i}-\mathrm{SiO}_{2} / \mathrm{n}-\mathrm{ZnO}$ heterojunctions based on hydrothermal ZnO nanorods. Mater. Sci. Semicond. Process. 2017, 66, 232-240. [CrossRef]

14. Chen, C.H.; Lee, C.T. High detectivity mechanism of ZnO-based nanorod ultraviolet photodetectors. IEEE Photonics Technol. Lett. 2013, 25, 348-351. [CrossRef]

15. Farhat, O.F.; Halim, M.M.; Ahmed, N.M.; Oglat, A.A.; Abuelsamen, A.A.; Bououdina, M.; Qaeed, M.A. A study of the effects of aligned vertically growth time on $\mathrm{ZnO}$ nanorods deposited for the first time on Teflon substrate. Appl. Surf. Sci. 2017, 426, 906-912. [CrossRef]

16. Zhang, H.; Babichev, A.V.; Jacopin, G.; Lavenus, P.; Julien, F.H.; Yu Egorov, A.; Zhang, J.; Pauporté, T.; Tchernycheva, M. Characterization and modeling of a $\mathrm{ZnO}$ nanowire ultraviolet photodetector with graphene transparent contact. J. Appl. Phys. 2013, 114, 234505. [CrossRef] 
17. Wu, J.-K.; Chen, W.-J.; Chang, Y.H.; Chen, Y.F.; Hang, D.-R.; Liang, C.-T.; Lu, J.-Y. Fabrication and photoresponse of $\mathrm{ZnO}$ nanowires/CuO coaxial heterojunction. Nanoscale Res. Lett. 2013, 8, 387. [CrossRef] [PubMed]

18. Dang, V.Q.; Trung, T.Q.; Duy, L.T.; Kim, B.-Y.; Siddiqui, S.; Lee, W.; Lee, N.-E. High-performance flexible ultraviolet (UV) phototransistor using hybrid channel of vertical ZnO nanorods and graphene. ACS Appl. Mater. Interfaces 2015, 7, 11032-11040. [CrossRef] [PubMed]

19. Dogar, S.; Khan, W.; Kim, S.-D. Ultraviolet photoresponse of ZnO nanostructured AlGaN/GaN HEMTs. Mater. Sci. Semicond. Process. 2016, 44,71-77. [CrossRef]

20. Li, Q.H.; Gao, T.; Wang, Y.G.; Wang, T.H. Adsorption and desorption of oxygen probed from ZnO nanowire films by photocurrent measurements. Appl. Phys. Lett. 2005, 86, 123117. [CrossRef]

21. Ambacher, O.; Smart, J.; Shealy, J.R.; Weimann, N.G.; Chu, K.; Murphy, M.; Schaff, W.J.; Eastman, L.F.; Dimitrov, R.; Wittmer, L.; et al. Two-dimensional electron gases induced by spontaneous and piezoelectric polarization charges in N- and Ga-face AlGaN/GaN heterostructures. J. Appl. Phys. 1999, 85, 3222-3233. [CrossRef]

22. Ture, E.; Brückner, P.; Quay, R.; Ambacher, O. Enhancement-Mode AlGaN/GaN FinFETs With High On/Off Performance in $100 \mathrm{~nm}$ Gate Length. In Proceedings of the 11th European Microwave Integrated Circuits Conference Enhancement-Mode; EuMA, London, UK, 3-4 October 2016; pp. 61-64.

23. Chang, S.-B.; Chae, H.U.; Kim, H.-S. Structural, optical, electrical and morphological properties of different concentration sol-gel $\mathrm{ZnO}$ seeds and consanguineous $\mathrm{ZnO}$ nanostructured growth dependence on seeds. J. Alloys Compd. 2017, 729, 571-582.

24. Khan, W.; Ajmal, H.; Khan, F.; Huda, N.; Kim, S.-D. Induced photonic response of ZnO Nanorods Grown on oxygen plasma-treated seed crystallites. Nanomaterials 2018, 8, 371. [CrossRef] [PubMed]

25. Yoon, Y.C.; Park, K.S.; Kim, S.-D. Effects of low preheating temperature for ZnO seed layer deposited by sol-gel spin coating on the structural properties of hydrothermal ZnO nanorods. Thin Solid Films 2015, 597, 125-130. [CrossRef]

26. Alenezi, M.R.; Henley, S.J.; Silva, S.R.P. On-chip fabrication of high performance nanostructured ZnO UV detectors. Sci. Rep. 2015, 5, 8516. [CrossRef] [PubMed]

27. Talib, R.A.; Abdullah, M.J.; Al-Salman, H.S.; Mohammad, S.M.; Allam, N.K. ZnO nanorods/polyaniline heterojunctions for low-power flexible light sensors. Mater. Chem. Phys. 2016, 181, 7-11. [CrossRef]

28. Willander, M.; Nur, O.; Sadaf, J.R.; Qadir, M.I.; Zaman, S.; Zainelabdin, A.; Bano, N.; Hussain, I. Luminescence from zinc oxide nanostructures and polymers and their hybrid devices. Materials 2010, 3, 2643-2667. [CrossRef]

29. Iqbal, M.; Ko, P.-S.; Kim, S.-D. Hot-electron reliability improvement using perhydropolysilazane spin-on-dielectric passivation buffer layers for AlGaN/GaN HEMTs. Curr. Appl. Phys. 2014, 14, 1099-1104. [CrossRef]

30. Alim, M.A.; Rezazadeh, A.A.; Gaquiere, C. Anomaly and threshold voltage shifts in GaN and GaAs HEMTs over temperature. In Proceedings of the 12th European Microwave Integrated Circuits Conference (EuMIC), Nuernberg, Germany, 8 October 2017; pp. 33-36.

31. Lee, M.L.; Sheu, J.K.; Lin, S.W. Schottky barrier heights of metal contacts to n-type gallium nitride with low-temperature-grown cap layer. Appl. Phys. Lett. 2006, 88, 032103. [CrossRef]

32. Cho, S.J.; Dogan, S.; Sabuktagin, S.; Reshchikov, M.A.; Johnstone, D.K.; Morkoc, H. Surface band bending in as-grown and plasma-treated n-type GaN films using surface potential electric force microscopy. Appl. Phys. Lett. 2004, 84, 3070-3072. [CrossRef]

33. Im, K.S.; Won, C.H.; Jo, Y.W.; Lee, J.H.; Bawedin, M.; Cristoloveanu, S.; Lee, J.H. High-performance GaN-based nanochannel FinFETs With/Without AlGaN/GaN heterostructure. IEEE Trans. Electron Devices 2013, 60, 3012-3018. [CrossRef]

34. Janotti, A.; van de Walle, C.G. Fundamentals of zinc oxide as a semiconductor. Rep. Prog. Phys. 2009, 72, 126501. [CrossRef]

35. Khan, F.; Khan, W.; Kim, J.H.; Huda, N.U.; Salman Ajmal, H.M.; Kim, S.-D. Oxygen desorption kinetics of ZnO nanorod-gated AlGaN/GaN HEMT-based UV photodetectors. AIP Adv. 2018, 8, 075225. [CrossRef]

36. An, Q.; Meng, X.; Xiong, K.; Qiu, Y. Self-powered ZnS nanotubes/Ag nanowires MSM UV photodetector with high on/off ratio and fast response speed. Sci. Rep. 2017, 7, 4885. [CrossRef] [PubMed] 
37. Brancaleon, L.; Lin, G.; Kollias, N. The in vivo fluorescence of tryptophan moieties in human skin increases with UV exposure and is a marker for epidermal proliferation. J. Investig. Dermatol. 1999, 113, 977-982. [CrossRef] [PubMed]

38. Al-Hardan, N.H.; Abdul Hamid, M.A.; Ahmed, N.M.; Jalar, A.; Shamsudin, R.; Othman, N.K.; Kar Keng, L.; Mohammed, S.M. A study on the UV photoresponse of hydrothermally grown zinc oxide nanorods with different aspect ratios. IEEE Sens. J. 2015, 15, 6811-6818. [CrossRef]

39. Bai, Z.; Yan, X.; Chen, X.; Zhao, K.; Lin, P.; Zhang, Y. High sensitivity, fast speed and self-powered ultraviolet photodetectors based on ZnO micro/nanowire networks. Prog. Nat. Sci. Mater. Int. 2014, 24, 1-5. [CrossRef]

40. Agrawal, J.; Dixit, T.; Palani, I.A.; Ramachandra Rao, M.S.; Singh, V. Fabrication of high responsivity deep UV photo-detector based on Na doped ZnO nanocolumns. J. Phys. D Appl. Phys. 2018, 51, 185106. [CrossRef]

41. Hen, H.A.O.C.; Inzhou, X.M.A.; Hang, J.I.Z.; Iuguo, Q.L.I.; Iu, H.U.L.; Hen, Z.U.C.; Hu, G.U.C.; Hu, S.H.C. Avalanche solar blind photodetectors with high responsivity based on $\mathrm{MgO} / \mathrm{MgZnO}$ heterostructures. Opt. Mater. Express 2018, 8, 785-793.

42. Khan, M.A.; Kuznia, J.N.; Olson, D.T.; Van Hove, J.M.; Blasingame, M.; Reitz, L.F. High-responsivity photoconductive ultraviolet sensors based on insulating single-crystal GaN epilayers. Appl. Phys. Lett. 1992, 60, 2917-2919. [CrossRef]

43. Bhardwaj, R.; Sharma, P.; Singh, R.; Gupta, M.; Mukherjee, S. High responsivity $\mathrm{Mg} \times \mathrm{Zn}_{1-\mathrm{x}} \mathrm{O}$ based ultraviolet photodetector fabricated by dual ion beam sputtering. IEEE Sens. J. 2018, 18, 2744-2750. [CrossRef]

44. Weng, W.Y.; Hsueh, T.J.; Chang, S.J.; Wang, S.B.; Hsueh, H.T.; Huang, G.J. A high-responsivity GaN nanowire UV photodetector. IEEE J. Sel. Top. Quantum Electron. 2011, 17, 996-1001. [CrossRef]

45. Bhattacharya, D.; Jha, N.K. FinFETs: From devices to architectures. In Digitally-Assisted Analog and Analog-Assisted Digital IC Design; Jiang, X., Ed.; Cambridge University Press: Cambridge, UK, 2015; Volume 2014, pp. 21-55. ISBN 9781316156148.

46. Crupi, F.; Alioto, M.; Franco, J.; Magnone, P.; Togo, M.; Horiguchi, N.; Groeseneken, G. Understanding the basic advantages of bulk FinFETs for sub- and near-threshold logic circuits from device measurements. IEEE Trans. Circuits Syst. II Express Briefs 2012, 59, 439-442. [CrossRef]

47. Wakejima, A.; Ando, A.; Watanabe, A.; Inoue, K.; Kubo, T.; Osada, Y.; Kamimura, R.; Egawa, T. Normally off AlGaN/GaN HEMT on Si substrate with selectively dry-etched recessed gate and polarization-charge-compensation $\delta$-doped GaN cap layer. Appl. Phys. Express 2015, 8, 026502. [CrossRef]

48. Im, K.-S.; Kim, R.-H.; Kim, K.-W.; Kim, D.-S.; Lee, C.S.; Cristoloveanu, S.; Lee, J.-H. Normally off single-nanoribbon $\mathrm{Al}_{2} \mathrm{O}_{3}$ GaN MISFET. IEEE Electron Device Lett. 2013, 34, 2012-2014. [CrossRef] 